MINI-SYMPOSIUM

\title{
Treating multivessel disease in the era of coated stents: conclusion
}

\author{
C Di Mario, H Griffiths
}

Heart 2004;90:1003. doi: 10.1136/hrt.2003.028829

$\mathrm{T}$ he worse long term outcome observed after percutaneous transluminal coronary angioplasty (PTCA) than after coronary artery bypass grafting (CABG) in patients at high risk of restenosis and disease progression such as diabetics, ${ }^{1-4}$ remind us that there is a substantial difference between treating a focal lesion with percutaneous coronary intervention and the use of arterial or vein grafts which bypass all proximal and mid segments of native coronary arteries. Interventionalists may argue that repair of the native arteries avoids the problem of accelerated disease of the bypass conduits and the occlusion of native arteries often seen years after implantation of vein grafts, situations difficult to handle both with surgery and angioplasty. Still, at least in the first few years after CABG and possibly much longer for arterial grafts, a bypass operation also prevents the acute events due to progression of disease in proximal and mid coronary segments arising from lesions that had no significant narrowing or only minor irregularities at the time of the initial treatment.

\section{DRUG COATED STENTS}

Progression of atherosclerosis cannot be addressed even with the best anti-restenotic devices. A drug coated stent may seal a lesion at risk of progression, but it is not reasonable to transform all of the coronary tree into a rigid metal tube to prevent plaque progression, and we still do not have reliable imaging or functional techniques to detect vulnerable plaques. The development of new lipid lowering drugs with high efficacy and low side effects is important since they may improve the long term outcome of a less invasive combined medical and percutaneous treatment strategy in patients with multivessel disease. ${ }^{5}$ With multivessel angioplasty these patients are unlikely to enjoy, as after surgery, a honeymoon period of a few years at very low risk of events even if they do not seek proper medical care and have limited adherence to risk factor control. Before treating multivessel disease patients with percutaneous techniques, we must be sure that the patients fully understand the importance of lifestyle changes and accept long term medical treatment with multiple drugs (antiplatelet and lipid lowering agents) and a programme of regular follow up visits and investigations.

\section{COSTS}

With this premise, and if the excellent angiographic result seen at two years after treatment with sirolimus eluting stents is maintained over time, the important step forward in terms of restenosis reduction with the use of drug eluting stents may justify percutaneous treatment of the majority of patients with multivessel disease. Reimbursement policy and cost of drug coated stents will also have a big impact on the speed this process will progress. Even if the cost per unit of these devices is reduced by the release of new drug eluting stents and market competition, the complexity of lesions treated must be taken into account by the health care providers to offer reimbursement policies more similar to those offered for surgical procedures in the same patients. In a retrospective review of 273 patients with multivessel disease who underwent bypass surgery at the Royal Brompton Hospital in 2002, percutaneous revascularisation with drug eluting stents was technically feasible in $75 \%$ of cases. However, this treatment would have involved lesions for which no data on long term results with drug eluting stents are available, and its one year cost would have been similar to the cost of bypass surgery. ${ }^{6}$ The switch from coronary surgery to interventional cardiology ultimately relies on the capacity of the interventional cardiologists to convince the medical community and health authorities, based on sound data from randomised trials, that percutaneous coronary intervention with drug eluting stents for multivessel disease is feasible, safe, clinically effective, and cost efficient.

Furthermore, it is not enough to increase the number and capacity of our interventional units: we must stop thinking of interventional cardiology as a simple practice every cardiologist can undertake, but instead train dedicated specialists able to cope with the complexity of the new tasks faced.

Correspondence to: Professor Carlo di Mario, Royal Brompton \& Harefield NHS Trust, Sydney Street, London SW3 6NP, UK; r.wall@rbh.nthames.nhs.uk

\section{REFERENCES}

1 Detre KM, Guo P, Holubkov, et al. Coronary revascularization in diabetic patients: a comparison of the randomized and observational components of the bypass angioplasty revascularization investigation (BARI). Circulation 1999;99:633-40.

2 King SB, Kosinski AS, Guyton RA, et al. Eight-year mortality in the Emory Angioplasty versus Surgery Trial. J Am Coll Cardiol 2000;35:116-21.

3 Abizaid A, Costa MA, Centemero M, et al. Clinical and economic impact of diabetes mellitus on percutaneous and surgical treatment of multivessel coronary disease patients. Insights from the arterial revascularization therapy study (ARTS) trial. Circulation. 2001;104: 533-8, 4.

4 Legrand V, Serruys $P$, Unger $F$, et al. Three-year outcome after coronary stenting versus bypass surgery for the treatment of multivessel disease. Circulation 2004; 109:114-20

5 Serruys PW, de Feyter P, Macaya C, et al. Fluvastatin for prevention of cardiac events following successful first percutaneous coronary intervention. A randomized controlled trial. JAMA 2002;287:3215-22.

6 Griffiths H, Di Mario C, Pepper J, et al. Feasibility and cost of treatment of surgical candidates with multivessel disease with drug eluting stents. Eur J Cardiothorac Surg (in press). 